\title{
Metamaterial Control of Hybrid Multifunctional High-Tc Superconducting Photonic Crystals for 1D Quasi-periodic Structure Potential Applications
}

\author{
Arafa H Aly, * (D, Doaa Mohamed ${ }^{a}$, M A Mohaseb M $^{a, b}$ \\ ${ }^{a}$ Beni-Suef University, Faculty of Science, TH-PPM Group, Physics department, Beni Suef, Egypt \\ ${ }^{b}$ Umm-Al-Qura University, Faculty of Applied Science, Department of Physics, Mecca, Saud Arabia
}

Received: December 30, 2019; Revised: April 28, 2020; Accepted: May 27, 2020

\begin{abstract}
In the present work, electromagnetic wave properties of the Fibonacci one-dimention photonic crystal (1DPC) structure consisting of double negative materials incorporated high Tc superconductor are theoretically investigated. It is found that the quasi-periodic structure created a photonic band gap as a periodic structure. We have calculated the transmittance spectra and noticed a wide band gap which can be controlled in it by the thickness of metamaterial, superconductor layer and incidence angle.Photonic band gap became more noticeable by increasing the thickness of the metamaterial and superconductor layers. The structure was affected by changing the incident angle and the band gap width increase with a noticeable shift to short wavelength region. Additionally, the photonic band gap shifted to longer wavelength value with increasing the operating tempeature. Furthermore, we have studied the pressure effects and we found the change in the location and width of photonic band gap.
\end{abstract}

Keywords: Optical properties, Pressure, High Tc superconductor, Photonic band gap, Fibonacii, Double negative materials

\section{Introduction}

Photonic crystals is artificial structures and attracted many researchers in the last years because their unique optical properties. They can generate the photonic band gap region where light cannot propagate in a similar way to the electronic band gaps in semiconductors ${ }^{1}$. Photonic crystals (PCs) has significant potential in optical communications and all modern photonic engineering. PCs composed of superconductor and dielectric materials attracted research interest in the recent past, due to the shorter dielectric losses, lower dispersion and wideband ${ }^{2-5}$. In 1968 , Vesselago ${ }^{6}$ predicted the negative index material (NIM) with a permittivity and permeability simultaneously turns to a negative value. This material is also known as double-negative (DNG) material that is an artificial composite, contrary to the usual double positive material. In addition to (DNG) material, a metamaterial can also be single negative material (SNG), that is, one of the two parameter $\varepsilon, \mu$ may be negative ${ }^{6-8}$. SNG material with negative permittivity called epsilon negative material (ENG), while a material with negative permeability called $\mathrm{Mu}$-negative material $(\mathrm{MNG})^{9,10}$. Superconducting and Metamaterial photonic crystals attract researchers interested in the past decades due to their unique properties ${ }^{11-16}$. Potential applications of metamaterials are various and include optical filters, medical devices, remote sensor detection and infrastructure monitoring, smart solar power management, crowd control, radomes, high-frequency battlefield communication and lenses for high-gain antennas ${ }^{17}$. In addition to the usual PCs which are

*email:arafa.hussien@science.bsu.edu.eg an aperiodic multilayer structure, there are quasi-periodic structures discovered in 1984. The quasi-periodic structure is nonperiodic structure, but they are constituted by a simple deterministic recursive value ${ }^{18}$. The quasi-periodic system can also possess forbidden frequency regions similar to the band gaps of a periodic $\mathrm{PCs}^{19-21}$. One of the quasi-periodic structures called the Fibonacci sequence (FS) has been investigated ${ }^{22,23}$. Recently, (FS) structure containing metamaterial has been studied, for which the zero n-gap ${ }^{24,25}$, the omnidirectional gap $^{26}$ and other unique phenomena were found, compared to the normal periodic PCs consisting of $\mathrm{MTMs}^{27}$. Based on the use of DNG material, the purpose of this paper is to study the transmission properties of Fibonacci photonic crystal consisting of metamaterial (DNG) and high Tc superconductor material theoretically.

\section{Theoretical Analysis}

Let us consider a finite $1 \mathrm{D}$ quasi-periodic structure which is based on Fibonacci generation consisting of two types of layers, A and B, arranged in a Fibonacci sequence. Here, $\mathrm{A}$ and $\mathrm{B}$ are considered metamaterials $(\mathrm{DNG})^{28}$, and high $\mathrm{Tc}$ superconductor material ( $\mathrm{HgBaCa}_{2} \mathrm{Cu}_{2} \mathrm{O}_{8+\delta}$ ). Figure 1 shows the fifth sequence $\mathrm{S} 5$, the sequences of Fibonacci is expressed as $S_{l+1}=\left\{S_{l} S_{l}-1\right\}$ for level $l \geq 1$. At $S_{0}=\{B\}, S_{1}=\{A\}$ the next sequences are $S_{2}=\{A B\}, S_{3}=\{A B A\}, S_{4}=\{A B A A B\}$, and so on. Here, it is considered that a TE wave is incident at an angle $\theta_{1}$ from the air with refractive index $n_{1}=1$. The first layer in the proposed structure possess a negative refractive index $\left(n_{2}\right)$, with effective $\varepsilon(f)$ and $\mu(f)$ given by ${ }^{28}$ : 


$$
\begin{gathered}
\varepsilon(f)=1+\frac{5^{2}}{0.9^{2}-f^{2}}+\frac{10^{2}}{11.5^{2}-f^{2}}, \\
\mu(f)=1+\frac{3^{2}}{0.902^{2}-f^{2}}
\end{gathered}
$$

Where $\mathrm{f}$ is the frequency measured in GHz. The second layer is a high Tc superconducting material which taken to be $\operatorname{Hg}(1223)$ with $\left(\mathrm{Tc}=134 \mathrm{~K} \text { and } \lambda_{0}=6100 \mathrm{~nm}\right)^{11,29}$, that possess refractive index $\left(n_{3}\right)$.

The Gorter Casimir Two-Fluid model was adopted to describe the electromagnetic response of the superconductor layer in the absence of an external magnetic field. The relative permittivity of superconductor material ${ }^{16}$.

$$
\varepsilon_{r}=1-\frac{1}{\omega^{2} \mu_{0} \varepsilon_{0} \lambda_{l}^{2}}
$$

Where the temperature dependance penetration depth is given by

$$
\lambda_{l}(T)=\frac{\lambda_{0}}{\sqrt{1-F(T)}}
$$

At Gorter-Casimir expression for $\mathrm{F}(\mathrm{T})$ is

$$
F(T)=\left(\frac{T}{T c}\right)^{4}
$$

Where $\lambda_{0}$ is the London penetration depth at zero temperature, and Tc is critical temperature. From Equation 3 refractive index of the superconductor layer will be $n_{3}=\sqrt{\varepsilon_{r}}$.

By including the effect of hydrostatic pressure in Hg-1223, Tc is modified ${ }^{30}$, and the thickness of the superconductor layer modified due to the compressibility constant, $\mathrm{Ka}=2.57 \times 10^{-3} \mathrm{GPa}^{-1}$. At $\lambda_{0}$ is constant, the relation between Tc and the applied pressure can be made in the following away:

$$
T c=q_{1}+q_{2} \cdot P+q_{3} \cdot P^{2}
$$

Where $q_{1}=134, q_{2}=2.009$, and $q_{3}=-4.194 \times 10^{-2}$ are constants due to the data from Takeshita et al. ${ }^{31}$, and $\mathrm{P}$ is the applied pressure.

By using Transfer matrix method we can calculate transmittance and reflectance of the quasi-periodic photonic ${ }^{32}$. Based on this theory the transmittance is expressible as:

$$
T=\left[2 G \div\left(\left(M_{11}+M_{12} G\right) G+M_{21}+M_{22} G\right)\right]^{2}
$$

with

$$
G=\sqrt{K_{0}^{2}-K_{x}^{2}} \div K_{0}=\cos \theta_{0}
$$

Where $\mathrm{G}$ in Equation 7 and 8 is the propagation matrix , and $K_{x}$ is X-component of the wave vector, $K_{0}$ is the free space wave vector.

the reflectance is given by

$$
R=1-T
$$

\section{Results and Discussion}

The transmittance spectra of the Fibonacci quasi-periodic metamaterial superconductor photonic crystal for different series/conditions are depicted in Figures 2-6. Figure 2 and 3 illustrate the difference between periodic and quasi-periodic photonic crystal structure with the same material. The main Fibonacci band gaps can be ascribed to local correlations in the form of periodic strings with the analogous wavelength and distribution of layers ${ }^{33}$. The difference between periodic and Fibonacci metamaterial superconductor photonic crystal are displayed in Figure 2 and 3 which is very close to the reported work of Trabelsi and Kanzari ${ }^{34}$. Figure 2 shows the transmittance spectra of periodic metamaterial photonic crystal for the $\mathrm{N}=55$ periods. The wide photonic band gap is

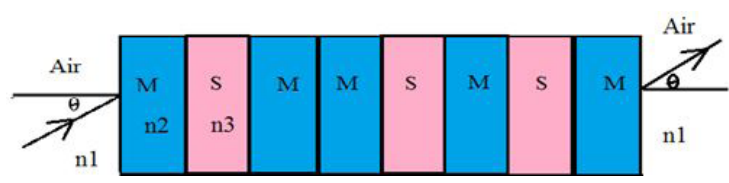

Figure 1. Schematic diagram of Fibonacci quasi-periodic 1DPC consisting of alternate the metamaterial $(\mathrm{M})$ and the superconducting material (S) under any incidence angle. The thicknesses of $\mathrm{M}$ and $\mathrm{S}$ are denoted by $\mathrm{d} 2$ and $\mathrm{d} 3$, respectively. And the corresponding refractive indices are separately indicated by $n 1, n 2$, and $n 3$, where $\mathrm{n} 1=1$ is taken

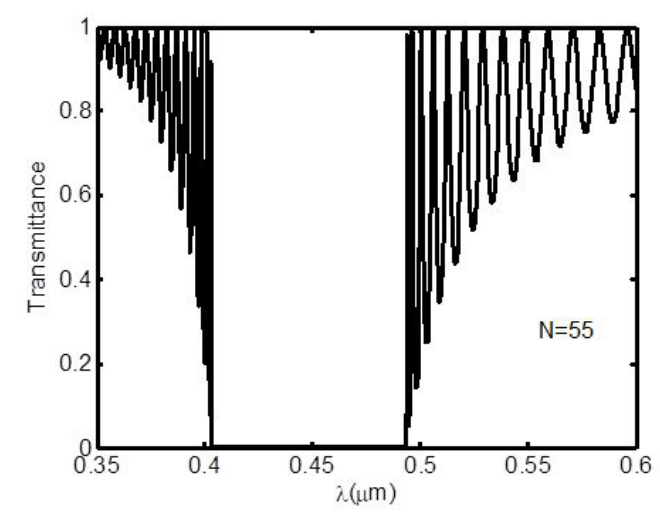

Figure 2. Transmittance spectra of periodic metamaterial superconductor photonic crystal for $\mathrm{N}=55$ periods.

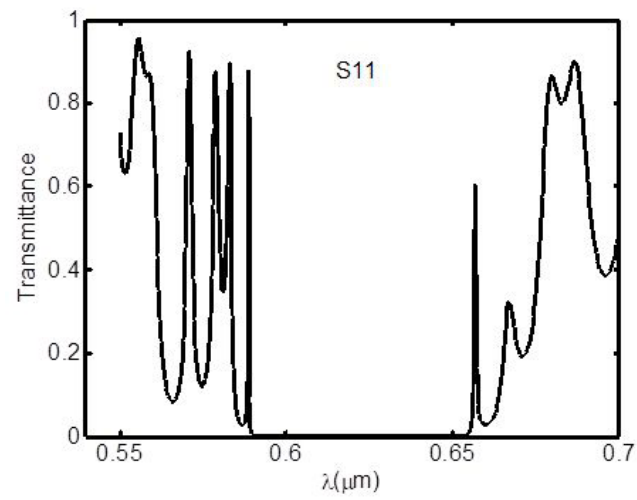

Figure 3. Transmittance spectrum of Fibonacci metamaterial superconductor photonic crystal for S11. 


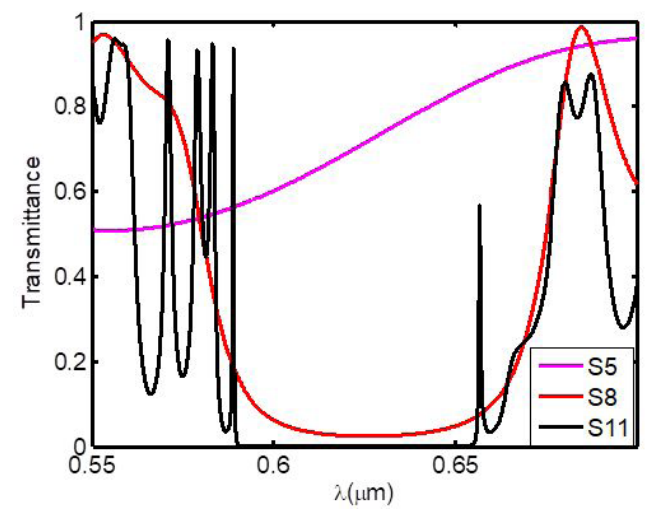

Figure 4. Transmittance spectra simulated on the quasi-periodic 1D PC structure with $\mathrm{d} 2, \mathrm{~d} 3$, and T fixed at $0.1,0.08 \mu \mathrm{m}$, and $100 \mathrm{~K}$, respectively, while varying the number of Fibonacci sequence from 5 to 11 .
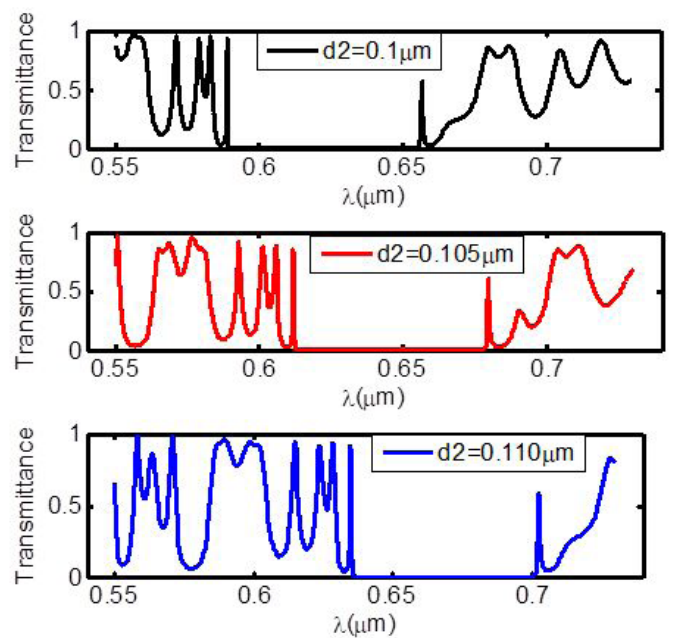

Figure 5. Transmittance spectra simulated on the quasi-periodic 1D PC structure with $\mathrm{d} 2$ varied to be $0.1,0.105$, and $0.110 \mu \mathrm{m}$ while $\mathrm{d} 3$ is fixed to be at $0.08 \mu \mathrm{m}$.
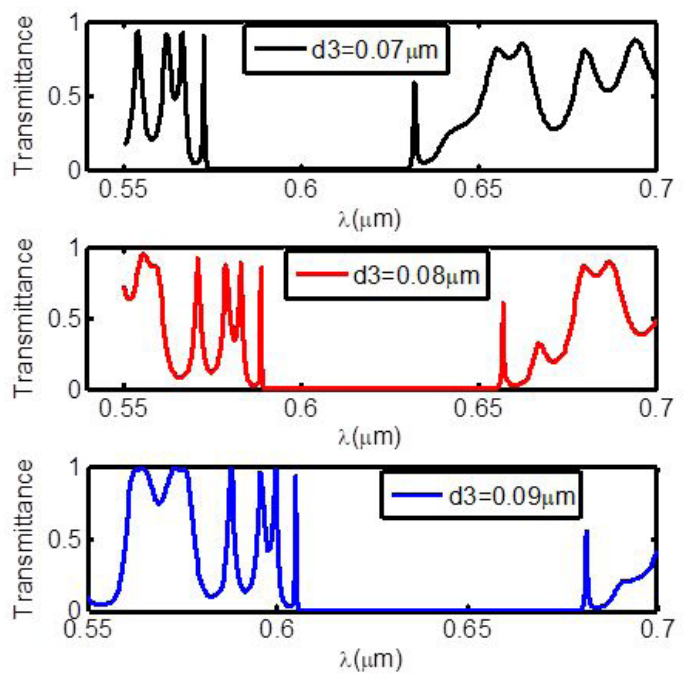

Figure 6. Transmittance spectra simulated on the quasi-periodic 1D PC structure with $\mathrm{d} 3$ varied to be $0.07,0.08$, and $0.09 \mu \mathrm{m}$ while $\mathrm{d} 2$ is fixed to be at $0.1 \mu \mathrm{m}$. noticed in a visible light region. In Figure 3 quasi-periodic structure for Fibonacci sequence S11, there are two defect peaks in the photonic band gap (PBG), and the (PBG) width increased with changing the optical range of the PBG. That is mean the electromagnetic waves will be more controlled by this structure.

The effect of Fibonacci levels of the proposed 1D metamaterial superconductor PCs structure is first evaluated as displayed in Figure 4. The number of levels was varied from 5 to 11 while fixing $\mathrm{d} 2, \mathrm{~d} 3$, and the ambient temperatures $\mathrm{T}$ to be $0.1 \mu \mathrm{m}(\mathrm{m}), 0.08 \mu \mathrm{m}(\mathrm{S})$, and $100 \mathrm{~K}$, respectively. By increasing the Fibonacci sequence to $\mathrm{S} 11$, it is noticed that the band gap edges become sharper, and the oscillation amplitude does not present a steady or gradual change on the contrary to the periodic metamaterial superconductor photonic crystal structure. Similar results were reported by $\mathrm{Wu}$ and $\mathrm{Gao}^{35}$. Figure 5 represents the simulated transmittance spectra of designed 1D Fibonacci metamaterial superconductor $\mathrm{PC}$, with $\mathrm{d} 2$ ranging from $(0.1 \mu \mathrm{m}$ to $0.110 \mu \mathrm{m})$ while fixed $\mathrm{d} 3$ at $(0.08 \mu \mathrm{m})$. The operating temperature is set to be $100 \mathrm{~K}$. When $\mathrm{d} 2$ is varied from 0.1 to $0.110 \mu \mathrm{m}$, the band gap edge shifted from $662 \mathrm{~nm}$ to $706 \mathrm{~nm}$. This behavior of increasing metamaterial thickness has corresponded Wu et al. ${ }^{36}$. In this reference by increasing the thickness of metamaterial that the authors ${ }^{36}$ used, we notice a small shift to long wavelength region and new three bands appeared for the main photonic band gap. But for our structure by increasing the thickness of metamaterial noticeable shifted to long wavelength region and several small gaps are observed in the left side of the main band gap which meaning that our structure is affected extensively by changing metamaterial thickness and could be useful in several optical applications as multichannel filters

Figure 6, illustrates the effect of increasing the thickness of high Tc superconductor layer from $(0.07-0.09) \mu \mathrm{m}$ by keeping the thickness of the metamaterial layer fixed at $\mathrm{d} 2=0.1 \mu \mathrm{m}$ and operating temperature at $\mathrm{T}=100 \mathrm{~K}$. The photonic band gap width is increased by increasing the thickness of the superconductor layer and shifted to long wavelength region corresponded by Ubeid et al. ${ }^{37}$. As a result, the simulation results in Figures 5 and 6 reveal that the quasi-periodic 1D metamaterial superconductor PC can act as a high-pass filter. By comparing with metallic PCs, the superconductor PCs can overcome the inherent loss issue coming from the metallic extinction coefficient.

Figure 7 shows that when the incident angle is increased from $\theta=0^{\circ}$ normal incidence to $\theta=20^{\circ}$, the bandwidth was increased and shifted to the shorter wavelength region with successive harmonic ordered stop band before the original band gap. This result is convergent to that of reported by Srivastava and Ojha ${ }^{38}$. In Figure 8, the effect of the change of operating temperature on the proposed structure is demonstrated. As shown in Figure 8 we present the transmittance spectrum of TE mode at $\theta=0^{\circ}$. By increasing the operating temperature from $\mathrm{T}=100 \mathrm{~K}$ to $450 \mathrm{~K}$, a noticeable change in the transmittance spectrum of the proposed structure was observed. The width of the PBG decreases with increasing the operating temperature and shifted to longer wavelength region which is similar to the ppublished results reported by $\mathrm{Wu}$ and $\mathrm{GaO}^{35}$. This result agrees with the electromagnetic variational theorem, low-frequency modes tend to concentrate 

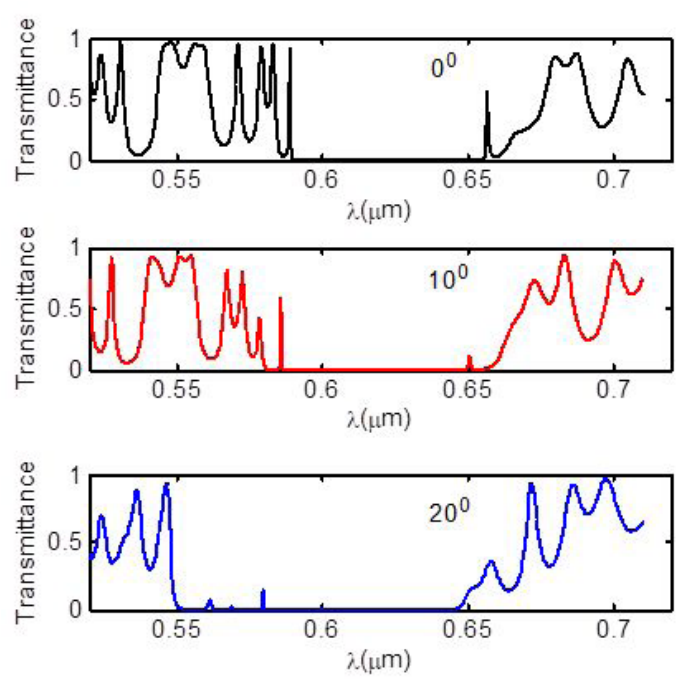

Figure 7. Transmittance spectra for quasi-periodic 1DPC at a different incident angle with fixing another parameter at $\mathrm{d} 2=0.1 \mu \mathrm{m}$, $\mathrm{d} 3=0.08 \mu \mathrm{m}$, and $\mathrm{T}=100 \mathrm{~K}$

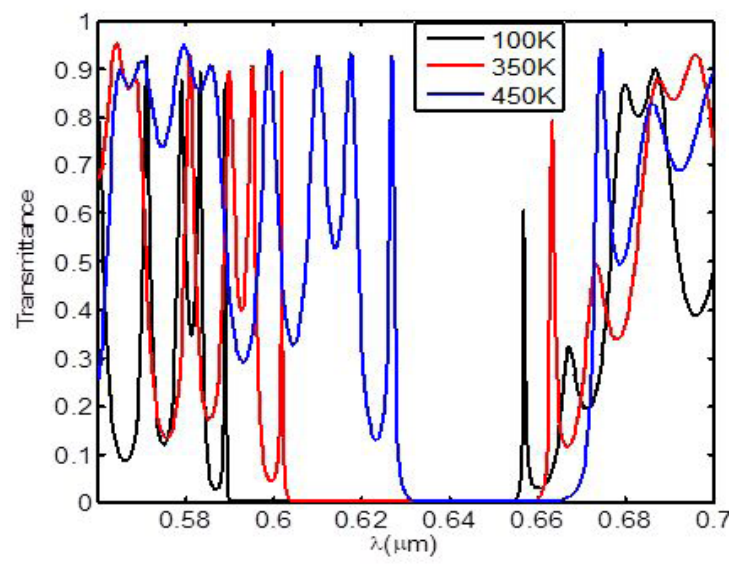

Figure 8. Transmittance spectra for quasi-periodic $1 \mathrm{DPC}$ at a different operating temperature with fixing the other parameters at $\mathrm{d} 2=0.1 \mu \mathrm{m}, \mathrm{d} 3=0.08 \mu \mathrm{m}$.

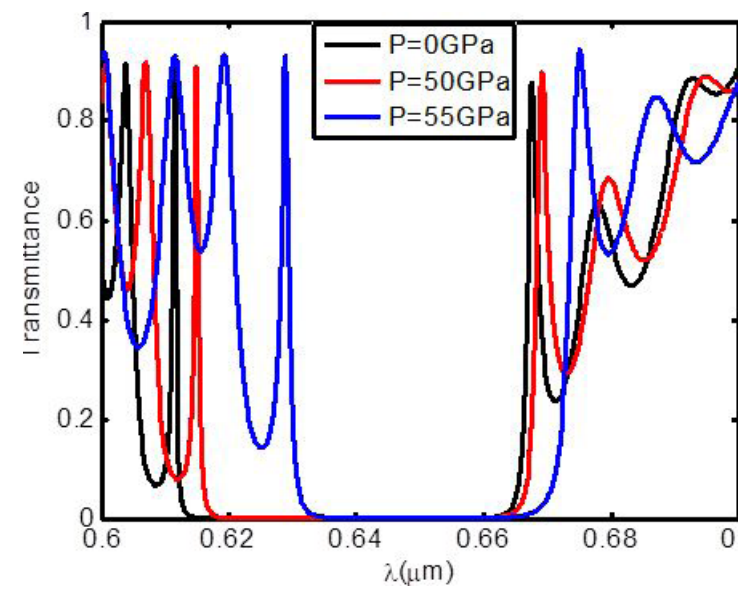

Figure 9. Transmittance spectra for quasi-periodic 1DPC at different values of applied pressure with fixing the other parameters at $\mathrm{d} 2=0.1 \mu \mathrm{m}, \mathrm{d} 3=0.08 \mu \mathrm{m}$, and $\mathrm{T}=400 \mathrm{~K}$. a great fraction of energy in the regions where the dielectric constants are the largest.

Finally, we study the effect of hydrostatic pressure on Hg-1223 material in 1D quasi -periodic metamaterial superconductor photonic crystal. Figure 9 illustrates the transmittance spectra for different values of applied hydrostatic pressure at $\mathrm{d} 2=0.1 \mu \mathrm{m}, \mathrm{d} 3=0.08 \mu \mathrm{m}$, and $\mathrm{T}=400 \mathrm{~K}$. In Figure 9, we have noted that when the pressure increases the resonant transmitted peak shifted to longest wavelength, while a wide PBG appears for the longest wavelength. This phenomenon can be used to fabricate pressure sensors, polychromatic filters, which are tunable by pressure or an omnidirectional high reflector. The tuning effect happened when we increase the applied pressure to be 55GPa. Furthermore, the band gap width decreases and shifted to long wavelength region this result is matched to that reported by Herrera et al. ${ }^{39}$. Figure 9 different from Figure 7 that found in Herrera ${ }^{39}$ at two points, the first one in our figure obtained the shift of band gap to short wave length region clearly and more than that shown in Herrera ${ }^{39}$. The second one obtained the band gap width increases with shift to short wave length region more than that shown in Herrera ${ }^{39}$. All these features meaning that our structure is more sensitive to pressure and pressure has a noticeable effect on the photonic band gap.

\section{Conclusion}

The transmittance properties of a Fibonacci quasi-periodic 1D metamaterial superconductor photonic crystal have been investigated theoretically based on Transfer matrix method. The transmittance spectra of periodic structure and quasi-periodic are studied and it is noticed that the band gap in quasi-structure became wider than which found in the periodic structure, and the oscillation amplitude did not present a steady or gradual change on the contrary to the periodic structure. The structure has a tunable photonic band gap which was tuned by the thicknesses of metamaterial, superconductor layers, the incident angle, operating temperature, and by the applied pressure. This structure can be used in a variety of applications such as sensors, pass-band filters, high reflection mirrors, and bolometers.

\section{References}

1. Raymond Ooi CH, Au Yeung TC, Kam CH, Lim TK. Photonic band gap in a superconductor-dielectric supper lattice. Phys Rev B. 2000;61(9):5920-3. http://dx.doi.org/10.1103/PhysRevB.61.5920.

2. Wu CJ, Chen MS, Yang TJ. Photonic band structure for a superconductor-dielectric superlattice. Physica C. 2005;432(34):133-9. http://dx.doi.org/10.1016/j.physc.2005.07.019.

3. Revathy V, Boopathi CS, Selvakumar K, Joseph Wilson KS, Taya $\mathrm{SA}$, Aly AH, et al. Nonlinear polarization in metal nanocomposite system based photonic crystals. Optik. 2019;176:78-84. http:// dx.doi.org/10.1016/j.ijleo.2018.09.038.

4. Aly AH, Ryu S-W, Hsu H-T, Wu C-J. THz transmittance in onedimensional superconducting nanomaterial-dielectric superlattice. Mater Chem Phys. 2009;113(1):382-4. http://dx.doi.org/10.1016/j. matchemphys.2008.07.123.

5. Aly AH, Ameen AA, ElSayed HA, Mohamed SH. Photonic crystal defective superconductor and black body radiations. Opt Quantum Electron. 2018;50(10):361. http://dx.doi.org/10.1007/s11082-0181632-8. 
6. Vesselago VG. The electrodynamics of substances with simultaneously negative values of $\varepsilon$ and $\mu$. Sov Phys Usp. 1968;10(4):10-509. http:// dx.doi.org/10.1070/PU1968v010n04ABEH003699.

7. Smith DR, Padilla WJ, Vier DC, Nemat-Nasser SC, Schultz S. Composite medium with simultaneously negative permeability and permittivity. Phys Rev Lett. 2000;84(18):4184-7. http://dx.doi. org/10.1103/PhysRevLett.84.4184.

8. Guo Z, Wu F, Xue C, Jiang H, Sun Y, Li Y, et al. Significant enhancement of magneto-optical effect in one-dimensional photonic crystals with magnetized epsilon-near-zero defect. J Appl Phys. 2018;124(10):103104. http://dx.doi.org/10.1063/1.5042096.

9. Alu A, Engheta N. Pairing an epsilon-negative slab with a munegative slab: resonance, tunneling and transparency. IEEE Trans Antenn Propag. 2003;51(10):2558-71. http://dx.doi.org/10.1109/ TAP.2003.817553.

10. Guo Z, Jiang H, Long Y, Yu K, Ren J, Xue C, et al. Photonic spin Hall effect in waveguides composed of two types of single-negative metamaterials. Sci Rep. 2017;7(1):7742. http://dx.doi.org/10.1038/ s41598-017-08171-y. PMid:28798319.

11. Li J, Zhou L, Chan CT, Sheng P. Photonic band gap from a stack of positive and negative index materials. Phys Rev Lett. 2003;90(8):083901. http://dx.doi.org/10.1103/PhysRevLett.90.083901. PMid:12633427.

12. Jiang H, Chen H, Li H, Zhang Y, Zi J, Zhu S. Properties of onedimensional photonic crystals containing single-negative materials. Phys Rev E Stat Nonlin Soft Matter Phys. 2004;69(6 Pt 2):066607. http://dx.doi.org/10.1103/PhysRevE.69.066607.PMid:15244764.

13. Aly AH, Malek C, Elsayed HA. Transmittance properties of a quasi-periodic one-dimensional photonic crystals that incorporate nanocomposite material. Int J Mod Phys B. 2018;32(21):1850220. http://dx.doi.org/10.1142/S021797921850220X.

14. Aly AH. Metallic and superconducting photonic crystal. J Supercond Nov Magn. 2008;21(7):421-5. http://dx.doi.org/10.1007/s10948008-0352-x.

15. Elsayed HA,Aly AH. Terahertz frequency superconductor-nanocomposite photonic band gap. Int J Mod Phys B. 2018;32(5):1850056. http:// dx.doi.org/10.1142/S021797921850056X.

16. Aly AH, Mohamed D. BSCCO/SrTiO3 one dimensional superconducting photonic crystal for many applications. J Supercond Nov Magn. 2015;18(6):1699-703. http://dx.doi.org/10.1007/s10948-015-2993-x.

17. Aly AH, Sabra W. Superconductor-semiconductor metamaterial photonic crystals. J Supercond Nov Magn. 2016;29(8):1981-6. http://dx.doi.org/10.1007/s10948-016-3478-2.

18. Brun M, Guenneau S, Movchan AB. Achieving control of in-plane elastic waves. Appl Phys Lett. 2009;94(6):61903. http://dx.doi. org/10.1063/1.3068491.

19. Chen M-S, Wu C-J, Yang T-J, FuhAY-G. Wave properties of Fibonacci sequence photonic crystal containing single-negative materials, solid stat communication. Solid State Commun. 2013;168:42-51. http://dx.doi.org/10.1016/j.ssc.2013.05.017.

20. Vyunishev AM, Pankin PS, Svyakhovskiy SE, Timofeev IV, Vetrov SY. Quasiperiodic one-dimensional photonic crystals with adjustable multiple photonic bandgaps. Opt Lett. 2017;42(18):3602. http:// dx.doi.org/10.1364/OL.42.003602.

21. Guo Z, Jiang H, Sun Y, Li Y, Chen H. Asymmetric topological edge states in a quasiperiodic Harperchain composed of split-ring resonators. Opt Lett. 2018;43(20):5142-5. http://dx.doi.org/10.1364/ OL.43.005142. PMid:30320840.

22. Sheng P. Scattering and localization of classical waves in random media. Singapore: World Scientific; 1990. http://dx.doi.org/10.1142/0565.

23. Fujiwara T, Kohmoto M, Tokihiro T. Multifractal wave functions on a Fibonacci lattice. Phys Rev B Condens Matter. 1989;40(10):74136. http://dx.doi.org/10.1103/PhysRevB.40.7413. PMid:9991159.
24. Bouazzi Y, Soltani O, Romdhani M, Kanzari M. Numerical investigation on the spectral properties of one-dimensional triadic-cantor quasi-periodic structure. Prog Electromagnetics Res. 2014;36:1-7. http://dx.doi. org/10.2528/PIERM14032602.

25. Li J, Zhao D, Liu Z. Zero- photonic band gap in a quasiperiodic stacking of positive and negative refractive index materials. Phys Lett A. 2004;332(5-6):461-8. http://dx.doi.org/10.1016/j. physleta.2004.10.003.

26. Bruno-Alfonso A, Reyes-Gómez E, Cavalcanti SB, Oliveira LE Band edge states of the $\langle\mathrm{n}\rangle=0$ gap of Fibonacci photonic lattices. Phys Rev A. 2008;78(3):035801. http://dx.doi.org/10.1103/ PhysRevA.78.035801.

27. Rahimi H, Roshan Entezar S. Wave propagation in double-period quasi-regular one-dimensional photonic crystals composed of single-negative metamaterials. Physica B. 2011;406(17):3322-7. http://dx.doi.org/10.1016/j.physb.2011.05.051.

28. Awad MA, Aly AH. Experimental and theoretical studies of hybrid multifunctional $\mathrm{TiO}_{2} / \mathrm{TiN} / \mathrm{TiO}_{2}$. Ceram Int. 2019;45(15):1903643. http://dx.doi.org/10.1016/j.ceramint.2019.06.145.

29. Tinkham M. Introduction to superconductivity. 2nd ed. New York: McGraw-Hill; 1996.

30. Yamamoto A, Takeshita N, Terakura C, Tokura Y. High pressure effects revisited for the cuprate superconductor family with highest critical temperature. Nat Commun. 2015;6(1):8990. http://dx.doi.org/10.1038/ncomms9990. PMid:26619829.

31. Takeshita N, Yamamoto A, Iyo A, Eisaki H. Zero resistivity above $150 \mathrm{~K}$ in $\mathrm{HgBa} 2 \mathrm{Ca} 2 \mathrm{Cu} 3 \mathrm{O} 8+\delta$ at high pressure. J Phys Soc Jpn. 2013;82(2):023711. http://dx.doi.org/10.7566/ JPSJ.82.023711.

32. Yeh P. Optical waves in layered media. New York: Wiley; 1988.

33. Ramanujam NR, El-Khozondar HJ, Dhasarathan V, Taya SA, Aly AH. Design of one dimensional defect based photonic crystal by composited superconducting material for bio sensing applications. Physica B. 2019;572:42-55. http://dx.doi. org/10.1016/j.physb.2019.07.051.

34. Trabelsi Y, Kanzari M. Omnidirectional reflection from deformed quasi-periodic one-dimensional photonic crystals in high frequency. Phys Procedia. 2009;2(3):947-51. http:// dx.doi.org/10.1016/j.phpro.2009.11.048.

35. Wu J-J, Gao J-X. Transmission properties of Fibonacci quasiperiodic one-dimensional superconducting photonic crystals. Optik. 2012;123(11):986-9. http://dx.doi.org/10.1016/j. ijleo.2011.07.015.

36. Wu MR, Wu CJ, Chang SJ. Near-infrared longitudinal plasmon polariton photonic gaps in a semiconductor metamaterial photonic crystal. Super and Micro. 2015;80:206-14. http:// dx.doi.org/10.1016/j.spmi.2014.12.035.

37. Ubeid MF, Shabat MM, Sid-Ahmed MO. Transmitted powers of waves through superconductor dielectric photonic crystal. Photonics Optoelectron. 2013;1(1):35-9. http://dx.doi.org/10.12720/ Inpo.1.1.35-39.

38. Srivastava SK, Ojha SP. enhancement of omnidirectional reflection bands in one-dimensional photonic crystals with left-handed materials. PIER. 2007;68:91-111. http://dx.doi. org/10.2528/PIER06061602.

39. Herrera AY, Calero JM, Porras-Montenegro N. Pressure, temperature, and thickness dependence of transmittance in a $1 \mathrm{D}$ superconductor-semiconductor photonic crystal. J Appl Phys. 2018;123(3):033101. http://dx.doi. org/10.1063/1.5009708. 